\title{
Simulation on the application of electric spring for reactive power compensation at load side
}

\author{
Ye-lin $\mathrm{Hu}$, Chun Yin, Hai-gang Zang, and Pan-pan Li \\ School of Electrical and Information Engineering \\ Anhui University of Science\&Technology \\ Huainan, China \\ Email: 136936873@qq.com
}

\begin{abstract}
Electric spring has a lot of advantages like simple control and without needing a lot of energy storage, which is applied to the load side by means of suppressing voltage fluctuation caused by the reactive power fluctuation. The simulation model with electric spring is built by using the software of Matlab/Simulink. Through observing the changed voltage waveform on power grids with or without electric spring, the simulation results show that the electric springs are capable of regulating voltage at the load side.
\end{abstract}

Keywords-stabilizing voltage; reactive power fluctuation ; reactive power compensation; electric spring

\section{INTRODUCTION}

Presently, many countries have proposed increasing the renewable energy power plants' share (e.g. wind power, solar energy, etc.) in the future power systems. Once the renewable energy as major energy in the future power systems, the conventional technology of regulating voltage will encounter various problems and the voltage quality of power grids will be lowered significantly [1-2]. In order to make the power grids adapt to this change, some scholars have presented that it is able to stabilize the fluctuation of the power grids caused by renewable energy that regulating the voltage of electric demand side. The traditional technology will have to be changed from centralized management of hundreds of plant units to the distributed control of millions of loads.

Electric spring, proposed by the team in the University of Hong Kong, is a new technology applied to the load side for stabilizing voltage fluctuation caused by renewable energy [3]. It is different from traditional reactive power compensation methods (such as, SVC, STATCOM) [4-5]. It is utilized to stabilize the voltage of critical load, through sacrificing the voltage stability of non-critical load. Critical load (sophisticated and medical electrical load, etc.) is sensitive to the change of voltage, which is connected to the power grids directly. Non-critical load (water heaters, lighting systems, etc.) is able to withstand voltage fluctuation, which is connected in series with electric spring, then connected to the power grids.

\section{BASIC PRINCIPLE OF ELECTRIC SPRING}

For stabilizing the voltage of the critical load, electric spring injects the compensated voltage to coupling point of the critical load and power grids. The compensated voltage and the

\author{
Zhao-quan Chen \\ Faculty of Physiscs \\ ST Petersburg State University \\ ST Petersburg, Russia
}

current through non-critical load are orthogonal (leading or lagging the current). When lagging, electric spring has a role in boosting voltage of critical load; when leading, electric spring plays a role in bucking voltage of critical load.

Schematic of electric spring can be simply expressed with regard a single-phase half-bridge inverter circuit, shown in Fig.1. Output voltage of electric spring is the sinusoidal wave, and this is guaranteed by the low-pass filter connected to the output side. PWM controls on or off of MOSFET, which is produced by the controlled circuit, and switching frequency of MOSFET is $20 \mathrm{kHz}$. The buffer circuit in Fig.1, which is the improved discharging suppressed RCD snubber circuit, can prevent the snubber capacitor and stray inductance of the main circuit to resonating effectively. The diode $D_{s}$ can restrain transient voltage if it is properly selected. $C_{s 1}$ and $C_{s 2}$ are $0.15 \mu \mathrm{F} . C_{s}$ is $0.25 \mu \mathrm{F} . R_{l}$ and $R_{2}$ are $60 \Omega$ [6].

External circuit of electric spring as shown in Fig.2, $V_{E S}$ is the injected voltage and $R_{f}, L_{f}(5 \mathrm{mH})$ and $C_{f}(13.2 \mu \mathrm{F})$ are parameters of the output filter [7].

From Fig. 1 and 2, the output voltage can be written as:

$$
V_{a}=\frac{V_{d c}}{2} P W M+\frac{V_{d c}}{2} \overline{P W M} .
$$

Let $T$ be the switching period and $n$ the order of harmonics. Decomposing $V_{a}$ into fundamental and high frequency harmonic components:

$$
V_{a}(t)=V_{1 a}+a_{n} \sin \left(\frac{2 n \pi}{T} t\right)+\sum_{n=1}^{\infty} b_{n} \cos \left(\frac{2 n \pi}{T} t\right) .
$$

$V_{a}$ averaged over one switching period can be written as:

$$
\begin{aligned}
\overline{V_{a}}(t)= & \frac{1}{T} \int_{0}^{t} V_{1 a}(t) d t+ \\
& a_{n} \sin \left(2 n \pi \frac{t}{T}\right)+\sum_{n=1}^{\infty} b_{n} \cos \left(2 n \pi \frac{t}{T}\right) .
\end{aligned}
$$

Since the effect of filter inductance, the current $I_{f}$ generated by imposed voltage would cause rapid reduction of the high harmonics. High harmonics are neglected, and then the current response If can be expressed as:

$$
L_{f} \frac{d I_{f}}{d t}+R_{f} I_{f}=\frac{1}{T} \int_{0}^{T} V_{1 a}(t) d t-V_{E S} .
$$




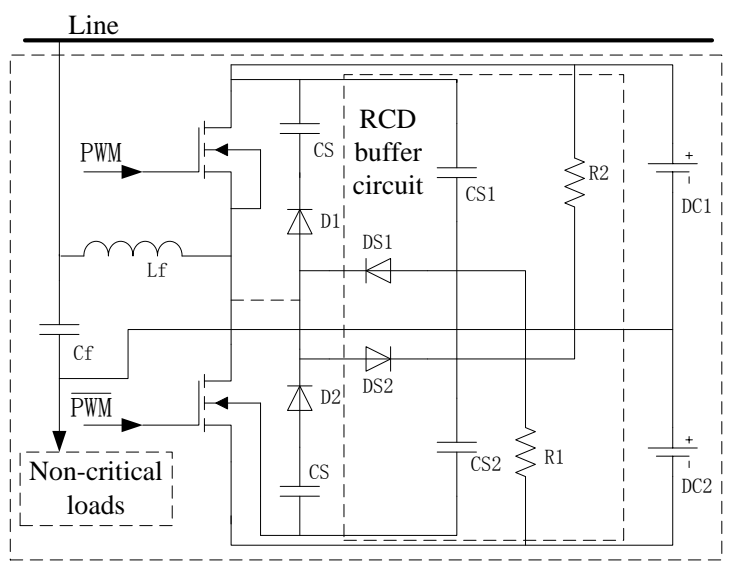

Fig. 1. Schematic of a single phase half bridge power inverter.

Hence, the fundamental voltage of the electric spring output can be written as:

$$
V_{1 \mathrm{a}}(t)=\frac{V_{d c}}{2} m(t) .
$$

Where $m(t)=M \sin (2 \pi 50 t+\theta)$ is the adjustment index. Removing the superbar for the reason for convenience, we can get the state-space averaged model as:

$$
\left[\begin{array}{c}
\frac{d I_{f}}{d t} \\
\frac{d V_{E S}}{d t} \\
\frac{d V_{d c}}{d t}
\end{array}\right]=\left[\begin{array}{cccc}
-\frac{R_{f}}{L_{f}} & \frac{m}{2 L_{f}} & -\frac{1}{L_{f}} & 0 \\
\frac{1}{C_{f}} & \frac{1}{R_{N L} C_{f}} & -\frac{1}{R_{N L} C_{f}} & 0 \\
0 & 0 & 0 & \frac{R_{f}}{C_{d c}}
\end{array}\right]\left[\begin{array}{c}
I_{f} \\
V_{d c} \\
V_{E S} \\
\frac{I_{f}^{2}}{V_{d c}}
\end{array}\right] .
$$

From (6), it can be seen that the state-space averaged model is nonlinear. Neglecting the DC link dynamics, we can express this with a set of linear time-invariant equations:

$$
\left[\begin{array}{c}
\frac{d I_{f}}{d t} \\
\frac{d V_{E S}}{d t}
\end{array}\right]=\left[\begin{array}{cc}
-\frac{R_{f}}{L_{f}} & -\frac{1}{L_{f}} \\
\frac{1}{C_{f}} & -\frac{1}{R_{N L} C_{f}}
\end{array}\right]\left[\begin{array}{c}
I_{f} \\
V_{E S}
\end{array}\right]+\left[\begin{array}{c}
\frac{1}{2 L_{f}} \\
\frac{1}{R_{N L} C_{f}}
\end{array}\right]\left[\begin{array}{c}
m V_{d c} \\
V_{c}
\end{array}\right] .
$$

\section{CONTROL OF ELECTRIC SPRINGS}

A diagram of electric spring controller model in Matlab/Simulink is set out in Fig.3. The dynamic changes of the DC link and the loss of electric spring are ignored in this model, wherefore electric spring only exchanges reactive power with the grids. It is essential that let the phase angle difference $\theta$ between $V_{E S}$ and $I_{O}$ be fixed to $\pi / 2$. The phase of $I_{O}$ is tracked by PLL. If the input of PI is negative, the phase of $I_{O}$ is added $-\pi / 2$, and if positive, added $\pi / 2$. The magnitude of $P W M$ depends on the PI output, which determines the value of $V_{E S}$. The sampling time of PI is 50ms. $K_{p}$ and $K_{i}$ are 15 and 750 respectively. The initial phase of $V_{E S}$ is set to $\pi / 2$ or $-\pi / 2$, because of the changes of the DC link being neglected.

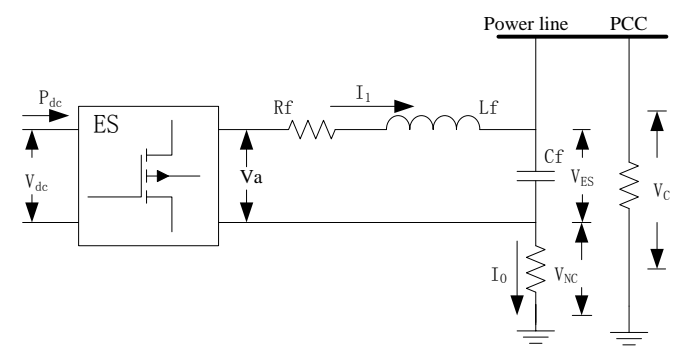

Fig. 2. The external circuit of electric spring.

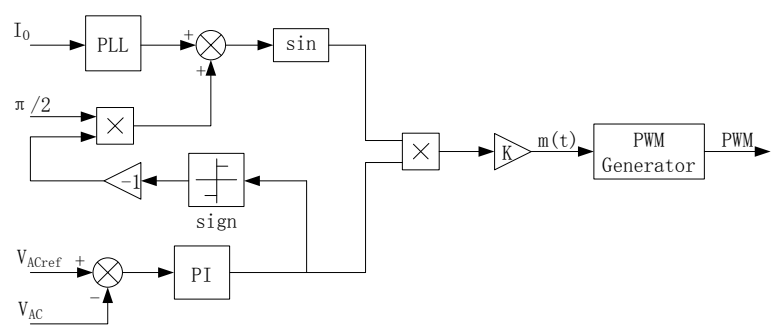

Fig. 3. Diagram of electric spring controller.

\section{Simulation OF ElECTRIC SPRINGS}

The overall simulation model of electric spring as shown in Fig.4, the frequency of Network is $50 \mathrm{~Hz}$. The resistance and inductance of the lines are $0.1 \Omega$ and $2.5 \mathrm{mH}$ respectively. The resistances of non-critical load and of critical load are $34.6 \Omega$ and $80.7 \Omega$ respectively. In the process of the simulation, let electric spring work in boosting bucking and random models.

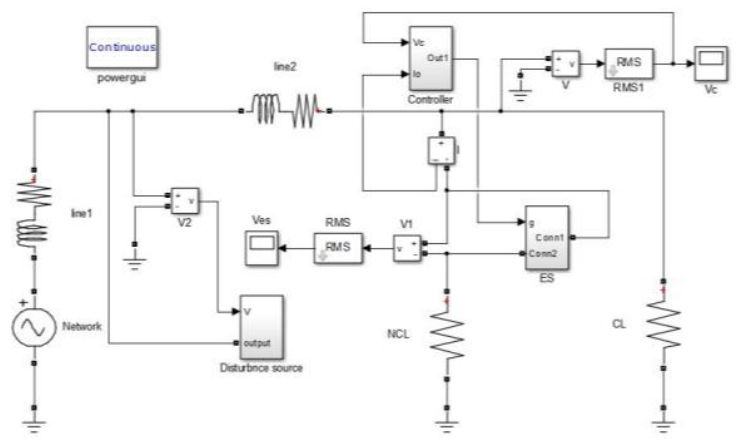

Fig. 4. Simulation model of Electric Spring.

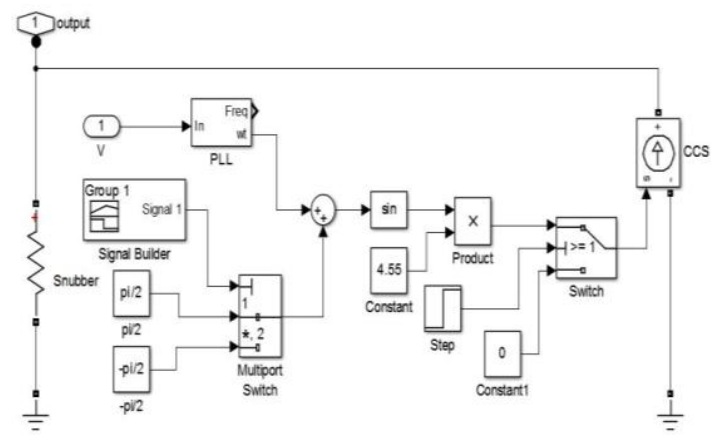

Fig. 5. Simulation model of disturbance source. 

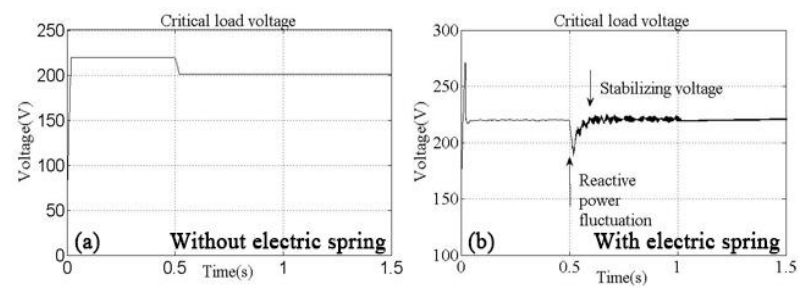

Fig. 6. Critical load voltage
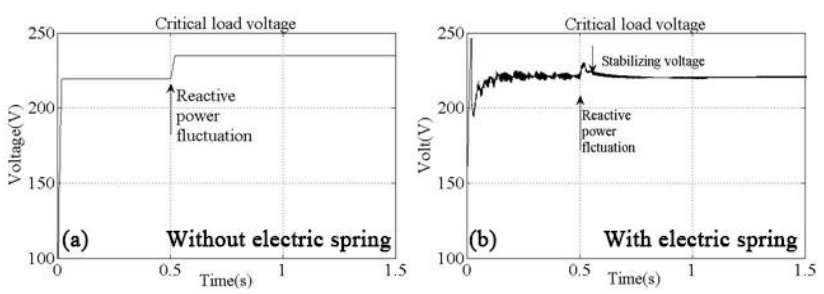

Fig. 7. Critical load voltage

\section{A. Disturbance Source Model}

A model of reactive power disturbance is a controlled current source, displayed in Fig.5. The Multiport Switch module chooses $+\pi / 2$ or $-\pi / 2$ to plus the phase of voltage at the output point, and the phase is tracked by PLL. So, the output current of the source and the voltage are orthogonal. The magnitude of the current is dependent on the value of Constant module. At the beginning time of the disturbance, a step signal is given to Switch module, and then the source starts to output the current to cause reactive power fluctuation. In the simulation, the value of reactive power is $1000 \mathrm{Var}$.

\section{B. Electric Spring Working in the Boosting Model}

In this model, $+\pi / 2$ is chosen by the Multiport Switch module, the current generated by the disturbance source leading the voltage of PCC. At $0.5 \mathrm{~s}$ a step signal is given to Switch module. The reactive power consumption is increased, and the critical load voltage is decreased. At the same time, electric spring begins to work to increase the voltage. The results of the simulation are presented in Fig.6. From (a), without electric spring, the voltage falls below $220 \mathrm{~V}$ after $0.5 \mathrm{~s}$ and cannot be recovered, but from (b), while with electric spring, the voltage can be increased to $220 \mathrm{~V}$ soon.

Voltage of electric spring voltage as shown in Fig.8 (a), at $0.5 \mathrm{~s}$, voltage of electric spring is changed to stabilize the voltage of the critical load to make the voltage be $220 \mathrm{~V}$.

\section{Electric Spring Working in the Bucking Model}

In this model, $-\pi / 2$ is chosen. At $0.5 \mathrm{~s}$, the reactive power consumption is decreased, and the critical load voltage is increased. At this moment, electric spring starts to work to reduce the voltage. The results of simulation are shown in Fig.7. As shown in (a), without electric spring, the voltage is higher than $220 \mathrm{~V}$ after $0.5 \mathrm{~s}$ and cannot be recovered, but as reported in (b), while with electric spring, the voltage can be decreased to $220 \mathrm{~V}$ quickly.

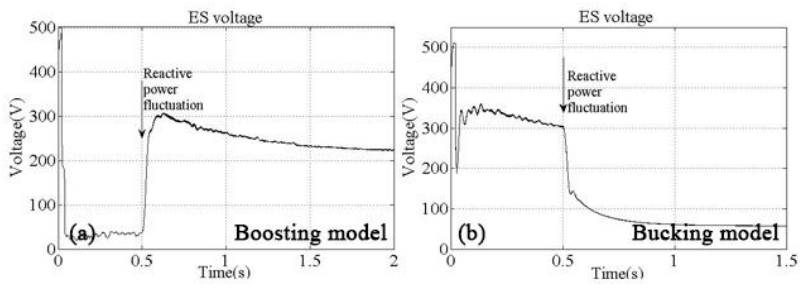

Fig. 8. Electric spring voltage
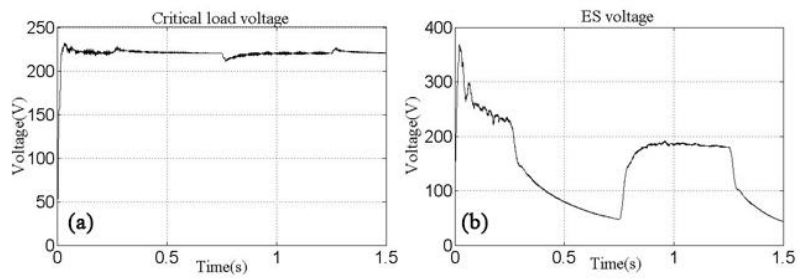

Fig. 9. Critical load voltage and electric spring

Voltage of electric spring voltage as shown in Fig.8 (b), at $0.5 \mathrm{~s}$, voltage of electric spring is changed to stabilize the voltage of the critical load to make the voltage be $220 \mathrm{~V}$.

\section{Electric Spring Working in the Random Model}

In this model, random variety of reactive power is imitated At $0.25 \mathrm{~s}$ and $1.25 \mathrm{~s}$, reactive power consumption is reduced to increase the voltage of the critical load; at $0.75 \mathrm{~s}$, reactive power consumption is increased to decrease the voltage of the critical load. The results are provided in Fig.9. After moments of reactive power disturbance, the voltage can be recovered to $220 \mathrm{~V}$ due to the work of electric spring.

\section{CONTROL OF ELECTRIC SPRINGS}

As mentioned above, electric spring is connected in series with non-critical load to balance voltage of critical load. It is generally applied to residential electricity side and other sides which require not much reactive power compensation. Reactive power compensation capacity of electric spring can be expressed as:

$$
Q_{E S}=V_{E S} \bullet I_{O}=V_{E S} \cdot \frac{V_{N C}}{R_{N C}} \cdot \sin \theta
$$

From (8) known, the compensated capacity $Q_{E S}$ is limited to the value of non-critical load, and the lager $R_{N C}$, the smaller $Q_{E S}$. Electric spring is applicable to the occasion that the proportion of non-critical load is low in the entire system, and also limited to this proportion.
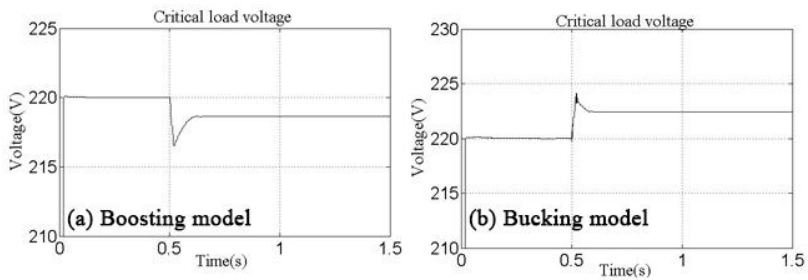

Fig. 10. Critical load voltage and electric spring 
Next, reversing critical load and non-critical load, so, the total load of the system isn't changed in the simulation. The value of reactive power disturbance isn't changed too. In this simulation, let electric spring work in boosting or bucking models, and results are presented in Fig.10.

The waveforms in Fig.10 indicate that, Even if the total load isn't changed and the value and nature of the reactive power fluctuation are same as before, the ability of electric spring for stabilizing the voltage is reduced significantly, due to that only the proportion between the non-critical load and the critical load is changed. This indicates that the applied field of electric spring is limited to the ratio between the noncritical load and the critical load.

\section{CONCLUSION}

The application of renewable energy can effectively alleviate the contemporary energy crisis and reduce the environmental pollution. But, it also generates considerable reactive power volatility to the grids and lowers voltage quality. By application of electric spring, these problems can be overcome.

The results of simulation above have shown that the voltage fluctuation can be stabilized effectively by electric spring. Even that the change of the DC link is tacked in to account, the effects of reactive power fluctuation can be eliminated. However, electric spring can only be applied in specific situations. The ratio of two loads and the value of reactive power fluctuation must be located in a certain range, so that, making electric spring subject to limitation on its application.

\section{ACKNOWLEDGMENT}

Work partially supported by the financial support from Natural Science Foundation of China (Grant No. 11575003), Natural Science Foundation in Anhui Province of China (Grant No. 1408085QA16, 1408085ME101), China Postdoctoral Science Foundation funded project (Grant No. 2014M551788), and Open-end Fund of State Key Laboratory of Advanced Electromagnetic Engineering and Technology (HUST). The authors would like to thank Professor Dr. Zhixiang Yin for their insightful discussions.

\section{REFERENCES}

[1] Xiao Xiang-ning, and Xu Yong-hai, "Power quality analysis and its development," Power System Technology, vol. 25, no. 3, pp. 67-69, Mar 2001.(in Chinese)

[2] LI Qiang, LI Feng-ting, FAN Yan-fang, et al., "Voltage influence of wind power integration," Electric Power, vol. 45, no. 34, pp. 15-18, Apr. 2012.(in Chinese)

[3] Shu Yuen(Ron) Hui, Chi Kwan Lee, and Felix F. Wu, "Electric springsa new smart grid technology," IEEE Trans. Smart Grid, vol. 3, no. 3, pp. 1552-1560, Sep. 2012.

[4] Deng Jia-ze, Wang Be, Huang Chong-xin, et al., "Reactive power compensation based on thyristor based STATCOM," Power System Technology, vol. 33, no. 1, pp. 48-51, Jan. 2009. (in Chinese)

[5] Siew-Chong Tan, Chi Kwan Lee, and S. Y. (Ron) Hui, "General steady analysis and control principle of electric spring with active and reactive power compensation," IEEE Trans. Power Electronics, vol. 28, no. 8, pp. 3958-3969, Aug. 2013.

[6] Tang Tantan, The research and revised design on buffer circuit of distribution staticsynchronous compensator, M.S. thesis, North China Electric Power University, Bao Ding, He Bei, 2012. (in Chinese)

[7] Nilanjan Ray Chaudhuri, Chikwan Lee, Balarko Chaudhuri, et al., "Dynamic modeling of electric springs," IEEE Trans. Smart Grid, vol. 5, no. 5, pp. 2450-2458, Sep. 2014. 